\title{
A NOTE ON THE CONSTRUCTION OF LARGE SET OF LATIN SQUARES WITH ONE ENTRY IN COMMON
}

\author{
CHIN-MEI FU*
}

\begin{abstract}
A latin square of order $n$ is an $n \times n$ array such that each of the integers $1,2,3, \cdots, n$ occurs exactly once in each row and each column. A large set of latin squares of order $n$ having only one entry in common is a maximum set of latin squares of order $n$ such that each pair of them contains exactly one fixed entry in common. In this paper, we prove that a large set of latin squares of order $n$ having only one entry in common has $n-1$ latin squares for each positive integer $n, n \geq 4$.
\end{abstract}

\section{Introduction and definitions}

A latin square of order $n$ is an $n \times n$ array such that each of the integers $1,2,3, \cdots, n$ (or any set of $n$ distinct symbols) occurs exactly once in each row and each column. A latin square $L=\left[l_{i, j}\right]$ is said to be idempotent provided that $l_{i, i}=i$. Two latin squares, $L=\left[l_{i, j}\right]$ and $M=\left[m_{i, j}\right]$ are said to have $k$ entries in common if there are exactly $k$ cells $(i, j)$ such that $l_{i, j}=m_{i, j}$. In [2], it was shown by H. L. Fu that there exists a pair of latin squares of order $n$ which have $k$ entries in common for each $n \geq 5$ and $k$ in $\left\{0,1,2, \cdots, n^{2}-7, n^{2}-6, n^{2}-4, n^{2}\right\}$. A large set of idempotend latin squares of order $n$ is a set containing maximum number of idempotent latin squares of order $n$ such that any two of them have $n$ entries in common and these $n$ entries are in the main diagonal. In [5], L. Terlinck and C. C. Lindner showed that a large set of idempotent latin squares

Received July 1, 1992; revised November 26, 1992.

* Research supported by NSC 81-0208-M-032-511. 
of order $n$ contains $n-2$ idempotent latin squares. We can extend this idea to the intersections of latin squares which are not necessarily idempotent. A large set of latin squares of order $n$ with $k$ entries in common is a maximum set of latin squares of order $n$ such that each pair of them contains exactly " fixed entries in common. Let $D(n, k)$ be the cardinality of a large set of laiin squares of order $n$ with $k$ entries in common. In [3], Fu showed that

$$
\begin{aligned}
D\left(n, n^{2}-4\right)= & 2, \text { for } n \geq 4, \\
D\left(n, n^{2}-6\right)= & D\left(n, n^{2}-7\right)=D\left(n, n^{2}-k\right)=2, \\
& \text { for } n \geq 6, k \in\left\{n^{2}-h \mid h=8,10,11,13,14\right\}, \\
D\left(n, n^{2}-9\right)= & 3, \text { for } n \geq 6 \text { and } \\
D\left(n, n^{2}-12\right)= & D\left(n, n^{2}-15\right)=3, \text { for } n \geq 8 .
\end{aligned}
$$

Then in [4], Hwang showed that $D(n, 1)=n-1$ for each $n \equiv 0$ or $2(\bmod$ 6 ). In this paper, by using a different technique, we prove that a large set of latin squares of order $n$ with one entry in common contains exactly $n-1$ latin squares, i.e. $D(n, 1)=n-1$, for each integer $n, n \geq 4$.

\section{Main Construction}

In [2], Fu showed the following theorem:

Theorem 2.1. There exist a pair of latin squares of order $v$ which have exactly $k$ entries in common, for each

(1) $k \in\{0,4\}$ when $v=2$;

(2) $k \in\{0,3,9\}$ when $v=3$;

(3) $k \in\{0,1,2,3,4,6,8,9,12,16\}$ when $v=4$; and

(4) $k \in\left\{0,1,2, \cdots, v^{2}-7, v^{2}-6, v^{2}-4, v^{2}\right\}$ when $v \geq 5$.

From Theorem 2.1 we find that for order 2 and 3 there is no large set of latin squares with one entry in common. Therefore in what follows we will consider the latin squares of order $n$ for $n \geq 4$.

It is easy to see that if two latin squares have one entry in common then we can use a row permutation and a column permutation such that this common 
entry is in the $(1,1)$-th cell. Without loss of generality, we let this entry be 1 , and denote $D(n, 1)$ by $D(n)$. Thus it is easy to get the following result.

Lemma 2.2. For any positive integer $n, D(n) \leq n-1$.

Proof. Since the (1,1)-th entry of all the latin squares we discuss should be 1 , the only way we can fill out the $(1,2)$-th entry of the large set of latin squares with one common entry must be one of the integers in $\{2,3, \cdots, n\}$. Since they must be different, therefore $D(n) \leq n-1$.

Next we will give a construction for those $D(n)$ latin squares of order $n$ with one fixed entry in common, for each $n \geq 4$.

Theorem 2.3. For any integer $n, n \geq 4$, there are $n-1$ latin squares of order $n$ such that any pair of them have one entry in common, i.e. $D(n)=n-1$.

Proof. (Since the proof will be by direct construction, it is helpful if the reader can look at the example following the proof step by step.) Let $M=\left[m_{i, j}\right]$ be an idempotent latin square of order $n-1$ based on the set $\{2,3, \cdots, n\}$. Let $P=\left[p_{i, j}\right]$ be defined as follows:

(1) $p_{1, j}=j$, for $j=1,2, \cdots, n$.

(2) $p_{i, 1}=i$, for $i=2,3, \cdots, n$.

(3) $p_{i+1, j+1}=m_{i, j}$, if $i \neq j, i, j=1,2, \cdots, n-1$, and

(4) $p_{i, i}=1$, for $i=1,2, \cdots, n$.

Then $P$ is a latin square based on the set $\{1,2, \cdots, n\}$. Let $F_{t}$ be a function from $\{2,3, \cdots, n\}$ to $\{1,2, \cdots, n\}$ defined as $F_{t}(j)=p_{t, j}$, for each $j=2,3, \cdots, n$ and $t=2,3, \cdots, n$. Then from $P$ and $F_{t}$ we can get $L(t)=\left[l(t)_{i, j}\right]$, for each $t \in\{2,3, \cdots, n\}$ defined as follows:

(1) $l(t)_{1,1}=l(t)_{t, t}=1$.

(2) $l(t)_{i, j}=F_{t}\left(p_{i, j}\right)$, for $i \neq j, i, j=2,3, \cdots, n$.

(3) $l(t)_{1, j}=F_{t}(j)$, for $j=2,3, \cdots, n$, and $j \neq t$.

(4) $l(t)_{i, 1}=l(t)_{1, i}$, for $i=2,3, \cdots, n$.

and

(5) $l(t)_{i, i}=p_{1, t}$, for $i \neq t, i=2,3, \cdots, n$. 
From the above construction, it is easy to check that $P$ is a latin square of order $n$ based on the set $\{1,2, \cdots, n\}$. By the properties of a latin square we know that $F_{t}$ is a one-to-one function from $\{2,3, \cdots, n\}$ to $\{1,2, \cdots, n\}$ for each $t$, and $F_{2}, F_{3}, \ldots, F_{n}$ are one-tu-one functions such that $F_{i}(x) \neq F_{j}(x)$ for each $i \neq j$ and $x \in\{2,3, \cdots, n\}$.

Next we will show that $L(t)$ is a latin square of order $n$, for each $t \geq 2$.

(1) For $i \neq j$ and $i \neq k$, where $i, j, k \in\{2,3, \cdots, n\}$, if $l(t)_{i, j}=l(t)_{i, k}$ then $F_{t}\left(p_{i, j}\right)=F_{t}\left(p_{i, k}\right)$, this implies that $p_{i, j}=p_{i, k}$. Thus $j=k$, since $P$ is a latin square.

(2) If $l(t)_{i, j}=l(t)_{i, i}$ then $F_{t}\left(p_{i, j}\right)=p_{1, t}$. From the definition of $F_{t}$, we know that $F_{t}\left(p_{i, j}\right)=p_{t, k}$ where $k=p_{i, j}$. Since $p_{1, t}=t=p_{t, 1}$, we get $p_{t, k}=p_{t, 1}$, implies $k=1$, i.e. $p_{i, j}=1=p_{i, i}$. Therefore $i=j$.

(3) If $l(t)_{1, j}=l(t)_{1, k}$ and $j, k>1$, then $F_{t}(j)=F_{t}(k)$, and hence $j=k$.

From (1), (2) and (3), we know that there is no symbol occurring twice in any row of $L(t)$. Similarly, we can prove that there is no symbol occurring twice in any column. Thus $L(t)$ is a latin square of order $n$. Since $F_{2}, F_{3}, \ldots, F_{n}$ are $n-1$ distinct functions, $L(2), L(3), \cdots, L(n)$ are disjoint latin squares except the $(1,1)$-th entry. Therefore there are $n-1$ latin squares of order $n$ such that any pair of them have one entry in common, for each $n \geq 4$.

Example. Using the construction above, we can construct a large set of latin squares of order 4 with one entry in common. Let $M$ be defined as follows:

$$
M=\begin{array}{lll}
2 & 4 & 3 \\
4 & 3 & 2 \\
3 & 2 & 4
\end{array}
$$

Then we can construct $P$ :

$$
P=\begin{array}{llll}
1 & 2 & 3 & 4 \\
2 & 1 & 4 & 3 \\
3 & 4 & 1 & 2 \\
4 & 3 & 2 & 1 \\
\hline
\end{array}
$$

By using $P$, we can generate three functions from $\{2,3,4\}$ to $\{1,2,3,4\}$ and 
obtain three latin squares, respectively.

$$
\begin{gathered}
F_{2}(2)=1, F_{2}(3)=4, F_{2}(4)=3 \\
L(2)=\left[\begin{array}{llll}
1 & 2 & 4 & 3 \\
2 & 1 & 3 & 4 \\
4 & 3 & 2 & 1 \\
3 & 4 & 1 & 2
\end{array}\right] \\
F_{3}(2)=4, F_{3}(3)=1, F_{3}(4)=2 \\
\left.L(3)=\begin{array}{llll}
1 & 4 & 3 & 2 \\
4 & 3 & 2 & 1 \\
3 & 2 & 1 & 4 \\
2 & 1 & 4 & 3
\end{array}\right] \\
F_{4}(2)=3, F_{4}(3)=2, F_{4}(4)=1 \\
L(4)=\begin{array}{llll}
1 & 3 & 2 & 4 \\
3 & 4 & 1 & 2 \\
2 & 1 & 4 & 3 \\
4 & 2 & 3 & 1
\end{array}
\end{gathered}
$$

Then it is easy to check that $L(2), L(3)$ and $L(4)$ have only one entry in common, mutually.

It seems that we can study this problem in a more general form by considering $k(\leq n)$ fixed common entries in the main diagonal of the latin squares of order $n \geq k$ such that $l_{i, i}=i, 1 \leq i \leq k$. The solution will naturally generalize the result in idempotent latin squares which is obtained in [5].

\section{Acknowledgement}

The comments of the referees helped the author to improve the representation of this paper. 


\section{References}

[1] J. Denes and A. D. Keedwell, "Latin Squares and their Applications," Academic, London, New York (1974).

[2] Hung-Lin Fu, "On the construction of certain types of latin squares having prescribed intersections," Ph. D. Thesis, Auburn University, 1980, December.

[3] Hung-Lin Fu, "More results on intersections of latin squares," Journal of Information and Optimization Sciences, Vol II, No. 3 (1990), pp. 525-535.

[4] Show-Ju Hwang, "Large set of latin squares with one entry in common," Master Thesis, Tamkang University, 1991.

[5] Luc Terlinck and C. C. Lindner, "The Construction of Large Sets of Idempotent Quasigroups," Eurpoean Journal of Combinatorial theory 9 (1988), pp. 83-89.

Department of Mathematics, Tamkang University, Tamsui, Taipei Shien, Taiwan, Republic of China. 